\title{
A Demanda de Trabalho das Empresas de Serviços TIC no Brasil, 2006 a 2013
}

\author{
Lucas Rodrigues Azambuja* \\ Daniel Gustavo Mocelin**
}

\section{Resumo}

Este artigo centra sua análise na demanda no mercado de trabalho tendo em vista o contexto de mudanças tecnológicas e socioeconômicas em razão do surgimento da chamada economia informacional. Empreendeu-se uma análise estatística descritiva, que compara a demanda das empresas de serviços considerados paradigmáticos da economia informacional, isto é, os serviços diretamente relacionados às tecnologias da informação e telecomunicação (TIC), com o setor de serviços como um todo. Essa análise utiliza dados da base RAIS para o Brasil entre os anos de 2006 a 2013. A análise teve como objetivo estabelecer algumas características da demanda por trabalho nesses ramos de atividades das TIC para guiar futuros estudos sobre a realidade laboral da economia informacional no Brasil. De modo geral, constatou-se que o setor de serviços TIC possui realidades laborais e organizacionais heterogêneas entre seus diversos segmentos, variando conforme o grau de inserção desses segmentos nos processos mais diretamente ligados à competição via inovação tecnológica..

\section{Palavras-chave}

Demanda de trabalho. Economia informacional. Setor de serviços.

\begin{abstract}
IThis article focuses its analysis on the demand in the labor market considering the context of technological and socioeconomic changes with the rise of the so-called informational economy. We undertook a descriptive statistical analysis that compares the companies' demand of services considered paradigmatic in the information economy, that is, the services
\end{abstract}

\footnotetext{
* Lucas Rodrigues Azambuja é professor adjunto no Instituto Brasileiro de Mercado de Capitais (IBMEC) - Minas Gerais; Doutor em Sociologia pela Universidade de São Paulo (USP); e pesquisador associado ao grupo de pesquisa "Sociedade, Economia e Trabalho", vinculado à Universidade Federal do Rio Grande do Sul (UFRGS). E-mail: Ir.azambuja81@gmail.com.

**Daniel Gustavo Mocelin é professor adjunto da Universidade Federal do Rio Grande do Sul (UFRGS) e Doutor em Sociologia pela mesma universidade; e pesquisador associado ao grupo de pesquisa "Sociedade, Economia e Trabalho" (UFRGS). E-mail: daniel.mocelin@ufrgs.br.
} 
directly related to information and communications technologies (ICT), to the service sector as a whole. This analysis uses Brazil's RAIS (Social Information Annual List) basis of data between the years 2006 and 2013. The analysis aimed to establish some characteristics of the labor demand in these ICT branches of activities to guide future studies on the labor reality of the informational economy in Brazil. In general, it was found that the ICT service sector has heterogeneous labor and organizational realities among the various segments, varying according to the degree of integration of these segments in the processes more directly linked to competition through technological innovation.

\section{Keywords}

Labor demand. Informational economy. The service sector.

\section{Introdução}

Uma característica do mercado de trabalho é a alteração de sua configuração em função das transformações econômicas e da mudança tecnológica. $\mathrm{Na}$ literatura recente, há um consenso razoável de que competição de mercado, inovações tecnológicas, liberalização comercial são processos interativos que estão entre as principais características das atividades econômicas que mais se expandiram nas últimas décadas. Porém, tais processos muitas vezes são apontados como causas da precarização do trabalho e do emprego em diversas atividades econômicas. Estudos recentes permitem questionar conclusões precipitadas sobre os impactos do desenvolvimento capitalista sobre o emprego, sugerindo que o dinamismo econômico também pode melhorar as condições de trabalho e de emprego para os trabalhadores, em função de novas lógicas produtivas, práticas administrativas e mercados competitivos, características do modo de desenvolvimento informacional, meio que é demandante de trabalho mais qualificado, embora não dispense trabalho não qualificado (INFANTE, 1999; FERRANTI et al., 2002; DE NEGRI et al., 2005; DE NEGRI et al., 2006).

Castells (2000) reconhece a polarização do mercado de trabalho, especialmente no caso de economias desenvolvidas. Segundo o argumento do autor, as atividades laborais mais ricas em conteúdo e que demandam maior qualificação dos trabalhadores são essenciais para o desempenho de setores produtivos de ponta, em expansão na economia atual. Essa configuração do mercado de trabalho tem relação com a forma empresarial, 
que passa a ser predominante, a "empresa em rede", que gera e processa informações com grande eficiência e uso de tecnologias informacionais, estabelece relações entre prestadores de serviços e fornecedores e permite flexibilidade para potencializar a inovação, transformando os meios de produção tão rapidamente quanto mudam os objetos sob o impacto de rápida transformação cultural e dos padrões de consumo.

Estudos realizados tanto nos Estados Unidos quanto nos países da América Latina demonstraram que a elevação da competitividade e a adoção de novas práticas de gestão têm resultado no aumento da dispersão salarial em favor dos trabalhadores mais qualificados, cuja produtividade e remunerações são maiores. Especialmente no caso da América Latina, a abertura econômica teria barateado os bens de capital, favorecendo aquisição de equipamentos mais modernos pelas empresas, que exigem trabalho mais qualificado no seu manuseio, eliminando trabalho menos qualificado e afetando a demanda e as remunerações em ocupações inferiores. (INFANTE, 1999; HUALDE; SERRANO, 2005).

É recorrente na literatura o argumento de que as características dos mercados de trabalho estão fortemente associadas com as novas dinâmicas da economia sob o paradigma informacional. Ferranti et al. (2002) observam que da mesma forma que a adoção de novas tecnologias demanda maior nível de capacitação dos empregados e os postos de trabalho demandariam mais dos trabalhadores em termos de capital humano, a competição estimula a adoção de novas tecnologias e estas, por sua vez, requereriam melhor capacitação e atualização. Essa dinâmica leva os autores a observar também que nos setores liberalizados os salários se mantêm em melhores condições que em setores mais protegidos, muito regulados e com formação de monopólios. No mesmo sentido, Centeno et al. (2007) destacam que o crescimento da produtividade nos mercados competitivos acaba sendo alcançado, principalmente, por meio da substituição de empresas pouco produtivas por outras mais produtivas e pela substituição de postos de trabalho pouco eficientes. Os autores concordam com a ideia de que a maior produtividade se traduz em salários mais altos e em mais empregos quando o ambiente concorrencial promove o uso de trabalho qualificado como fator produtivo essencial.

Nesta breve revisão da literatura, verificamos a tendência dos estudos em centrar sua análise no "lado da oferta" do mercado de trabalho, isto é, em compreender os impactos e tendências das transformações recentes da economia capitalistas nas ocupações tendo em conta perfil de trabalhadores 
e de empregos. Em contraste, o presente estudo centra sua análise no "lado da demanda" no mercado de trabalho, ou seja, procura fazer uma análise descritiva inicial sobre algumas características do comportamento da demanda de trabalho de empresas situadas em serviços considerados paradigmáticos da economia informacional, isto é, os serviços diretamente relacionados às tecnologias da informação e telecomunicação. O objetivo é que este estudo forneça evidências e conclusões iniciais a serem verificadas e aprofundadas em futuras pesquisas.

O artigo está dividido em: 1) discussão sobre a definição de firmas de serviços de TIC e sua atuação no mercado de trabalho; 2) considerações metodológicas; 3) análise dos dados RAIS; 4) considerações finais sobre as características e tendências que se destacam na demanda de trabalho das empresas de serviços TIC no Brasil.

\section{Empresas de Serviços de TIC: definições e o seu lugar no Mercado de Trabalho}

As tecnologias da informação e comunicação (TIC), principalmente nas últimas décadas, têm sido marcadas por intensas e rápidas inovações e por sua integração a outras tecnologias, como as biológicas e as audiovisuais. Isso faz com que seja uma tarefa complexa estabelecer uma noção de TIC. A definição de Castells é, nesse sentido, ilustrativa com relação ao caráter integrado dessas tecnologias: "Entre as tecnologias da informação, incluo [...] o conjunto convergente de tecnologias em microeletrônica, computação (software e hardware), telecomunicações/radiodifusão, e optoeletrônica" (2000, p. 67, grifos do autor). O autor ainda considera como parte das TIC a engenharia genética, pois ela lida com os códigos de informação da matéria viva e por ter interagido com a eletrônica e informática ao longo da década de 1990.

É ainda em função dessa convergência com outras tecnologias que, frequentemente, tecnologias da informação e da telecomunicação, apesar de diferentes, são definidas numa mesma categoria, as TIC ${ }^{1}$. Nesse sentido,

\footnotetext{
${ }^{1} \mathrm{O}$ aparelho celular é talvez o símbolo mais expressivo dessa convergência. Num mesmo dispositivo há funções de telefonia, Internet, televisão, vídeo, música, texto, jogos, GPS, etc. Tablets e Smatphones ampliam ainda mais a convergência tecnológica.
} 
A convergência configura-se, assim, como o conectivo tecnológico de base digital que possibilita a comunicação entre diversos tipos de aparelhos e equipamentos compreendidos no conjunto das TICs, permitindo a migração de dados, textos, sons e imagens entre diferentes aparelhos e equipamentos de informação e até mesmo a conjunção de diferentes formas de comunicação em um mesmo aparelho ou equipamento (SALDANHA, 2006; p. 65).

O IBGE (Instituto Brasileiro de Geografia e Estatística), na CNAE (Classificação Nacional de Atividades Econômicas) 2.0, estabeleceu o setor de "Informação e Comunicação" ${ }^{2}$, conforme tabela 1:

\section{Tabela 1 - Setor de TICs segundo CNAE 2.0}

Hierarquia
Seção: J Informação e Comunicação
DIVISÕES:
58 - Edição e edição integrada à impressão
59 - Atividades cinematográficas, produção de vídeos e de programas de televisão;
gravação de som e edição de música
60 - Atividades de rádio e de televisão
61 - Telecomunicações
62 - Atividades de serviços de tecnologia da informação
63 - Atividades de prestação de serviços de informação

Fonte: Comissão Nacional de Classificação.

Nota-se que essa definição se faz pela ótica dos produtos e serviços nos quais as TICs se materializam. Também que foram excluídas as atividades industriais de fabricação de máquinas e equipamentos de TIC ${ }^{3}$.

Diferenças de definições acontecem em razão de as TICs serem determinadas ou como um setor econômico, ou um grupo de produtos e serviços ou um paradigma tecnológico (EISCHEN, 2000). Essas diferentes "portas de entrada" para definição de TIC produzem, então, uma cacofonia conceitual que, nos parágrafos anteriores, apenas se arranhou a superfície.

\footnotetext{
${ }^{2}$ A CNAE 1.0 não apresentava um setor específico de informação e comunicação. Era preciso, então, construir uma classificação satélite na forma de agregados alternativos. Em IBGE (2009) podemos ver um exemplo desse esforço de classificação.

${ }^{3}$ Essas atividades constam na seção C (Indústria de Transformação), divisão 26 (Fabricação de equipamentos de informática, produtos eletrônicos e ópticos).
} 
Tendo em vista a centralidade das atividades de serviços nas discussões sobre as transformações recentes do capitalismo, elaboramos neste artigo uma noção de TIC para, principalmente, localizar empresas que representem casos-tipo na demanda de trabalho da nova economia. Assim sendo, elaborouse uma definição como um gênero de serviços que constituem a atividade-fim de certos grupos de empresas. A intenção, portanto, é alcançar uma definição que permita identificar os tipos de empresas que estão produzindo aqueles serviços que se constituem referências ubíquas nos processos que configuram o surgimento e funcionamento de uma nova etapa da economia capitalista. Portanto, nesta pesquisa compreendemos por TIC o conjunto de serviços que lidam com a produção, tratamento e/ou circulação da informação (seja na forma de texto, imagem, dado ou som) através de meios eletrônicos ou digitais.

Delimitar pelo critério de atividade-fim é ainda mais importante quando temos em vista os resultados de pesquisa de Hualde e Micheli (2009). Segundo esses autores, no segmento de serviços relacionados às tecnologias da informação se constituem duas realidades que, frequentemente, são tratadas indistintamente e amalgamadas dentro daquilo que se pode chamar de "setor econômico de tecnologias da informação", quais sejam: "serviços de tecnologia da informação" e os "serviços propiciados pelas tecnologias da informação". No primeiro estão situadas as firmas que têm como atividade-fim a prestação de serviços genuinamente de TI, como empresas de desenvolvimento de software. No segundo se constata a presença de empresas bastante distintas das do primeiro grupo, por exemplo, os centros de teleatendimento que prestam serviços de marketing, suporte técnico e/ou vendas.

A definição do setor de TIC elaborada neste artigo se mostra pertinente, pois através dela se podem distinguir essas duas realidades de segmentos do setor de serviços de tecnologia da informação, que, como demonstram Hualde e Micheli (2009), possuem um perfil diferente de mão de obra empregada do ponto de vista de níveis de remuneração, modalidade de contrato de trabalho, nível de escolaridade, etc. Portanto, se propõe examinar empresas que justamente são as que estão mais diretamente relacionadas com as tecnologias da informação, não somente porque utilizam essas últimas como ferramentas de trabalho e gestão, como também são essas tecnologias o que elas ofertam no mercado. Na CNAE 2.0, tal diferença transparece ao identificarmos que as atividades de teleatendimento nem mesmo estão classificados na seção J, "Setor de Informação e Comunicação", aparecem na seção N, denomina de "Atividades Administrativas e Serviços Complementares". 


\section{Procedimentos Metodológicos}

Neste artigo é feita uma análise quantitativa descritiva da evolução da demanda de trabalho no setor de TIC no que diz respeito às características das firmas, vínculos de trabalho utilizados e à força de trabalho por elas empregadas. Nosso objetivo não é testar hipóteses previamente formuladas e, sim, apresentar evidências que fornecem um panorama da demanda de trabalho no setor de serviços TIC, setor este que tem sido apontado como paradigmático na dinâmica das transformações econômicas recentes. Assim, nosso objetivo é trazer aspectos desse segmento do mercado formal de trabalho brasileiro, apontando para princípios conceituais e metodológicos que subsidiem futuras pesquisas. Para garantir um parâmetro de compreensão dos dados desse setor específico, elegemos por compará-los com o grande setor do qual essas atividades fazem parte, qual seja, o Setor de Serviços tal como é definido pelo IBGE com base na CNAE.

Utilizamos como fonte de dados a Relação Anual de Informações Sociais (RAIS) do Ministério do Trabalho e Emprego (MTE). A RAIS é um registro administrativo, de âmbito nacional, com periodicidade anual, obrigatório para todos os estabelecimentos públicos e privados. Ao contrário das pesquisas domiciliares cuja coleta tem objetivos estatísticos, a RAIS é feita com finalidades administrativas e, em razão dessa característica, apresenta limitações conceituais, porém tem caráter censitário. Essa fonte fornece informações do universo nacional de empresas com um ou mais vínculos de trabalho no ano-base, sendo que também podemos vislumbrar o número de estabelecimentos empresariais sem vínculos no ano, ou seja, a chamada declaração de "RAIS negativa". A RAIS compreende somente o chamado mercado formal de trabalho, não possibilitando vislumbrar vínculos $\mathrm{e}$ firmas que atuem de modo informal; e o MTE obtém esses dados através de formulários preenchidos pela própria empresa - então, distorções podem ocorrer. Sendo assim, consideram-se aqui os dados da RAIS como um proxy da demanda formal de trabalho do setor de TIC.

Para classificar o tipo de atividade exercida pela empresa, a RAIS utiliza a CNAE. Foi utilizada a CNAE 2.0 porque é a versão que define especificamente o setor de TICs e, também, porque os dados mais recentes da RAIS estão estruturados nessa versão.

Conforme já mencionado na Tabela 1, a CNAE inclui as atividades de conteúdo, isto é, serviços audiovisuais, rádio e televisão. Como nossa definição de setor TIC envolve apenas empresas cuja atividade-fim consiste em serviços 
diretamente relacionados à difusão mais intensa das TICs, escolhemos centrar nossa análise apenas nas divisões 61 (Telecomunicações), 62 (Atividades de serviços de tecnologia da informação) e 63 (Atividades de prestação de serviços de informação), as quais são amostra fiel do setor de atividade econômica em questão e categorias de padrão comparativo internacional. As três tabelas seguintes trazem a definição de cada uma dessas divisões da Seção J da CNAE 2.0:

Tabela 2 - Divisão dos Serviços Telecomunicações

\begin{tabular}{l} 
SEÇÃO \\
\hline J - Informação e Comunicação \\
DIVISÃO \\
61 - Telecomunicações \\
GRUPOS \\
611 - Telecomunicações por fio \\
612 - Telecomunicações sem fio \\
613 - Telecomunicações por satélite \\
614 - Operadores de televisão por assinatura \\
619 - Outras atividades de telecomunicações
\end{tabular}

Fonte: Comissão Nacional de Classificação.

Tabela 3 - Divisão dos Serviços de Tecnologia da Informação

Hierarquia
SEÇÃO
J - Informação e Comunicação
DIVISÃO:
62 - Atividades de serviços de tecnologia da informação
GRUPO
620 - Atividades de serviços de tecnologia da informação
CLASSES
6201-5 Desenvolvimento de Programas de Computador sob Encomenda
$6202-3$ Desenvolvimento e Licenciamento de Programas de Computador
Customizáveis
$6203-1$ Desenvolvimento e Licenciamento de Programas de Computador Não
Customizáveis
$6204-0$ Consultoria em Tecnologia da Informação
$6209-1$ Suporte Técnico, Manutenção e Outros Serviços em Tecnologia da
Informação

Fonte: Comissão Nacional de Classificação. 
Tabela 4 - Divisão de Prestação de Serviços de Informação

Hierarquia

SEÇÃO

J - Informação e Comunicação

DIVISÃO:

63 - Atividades de prestação serviços de informação

GRUPO

631 - Tratamento de dados, hospedagem na internet e outras atividades relacionadas 639 - Outras atividades de prestação de serviços de informação

Fonte: Comissão Nacional de Classificação.

Assim, nesta pesquisa, o setor de serviços TIC está delimitado como as atividades diretamente envolvidas em serviços de tecnologias da informação e comunicação, isto é, firmas pertencentes às divisões 61, 62 e 63 da seção J da CNAE 2.0. Como a adoção da CNAE 2.0 se refere somente aos dados da RAIS entre os anos de 2006 a 2013, optou-se por esse recorte temporal na análise que segue, evitando, assim, possíveis distorções nos dados em função de adaptações entre diferentes versões da CNAE.

A unidade de análise consiste na demanda da firma, porque esse é o objeto deste artigo. Assim, além das características das firmas de TIC, coletaramse dados que dizem respeito à frequência e intensidade da sua demanda, características dos vínculos e mão de obra por elas demandadas. Portanto, o interesse não é compreender o perfil dos trabalhadores e como eles circulam nesse mercado de trabalho, mas o comportamento da demanda das empresas de TIC. Por essa razão, nos dados que se seguem estão inclusos os vínculos ativos e inativos em 31 de dezembro do ano base. Poder-se-ia argumentar que, ao fazer isso, consideram-se duas ou mais vezes o mesmo trabalhador; entretanto, esse problema não se aplica, porque esta análise se estrutura para compreender a demanda e não a oferta de trabalho, ou seja, o que se está mensurando é a ação de demandar da firma de TIC no mercado de trabalho.

Entretanto, mais uma limitação da RAIS se revela neste ponto da demanda da firma como unidade de análise, a saber, a RAIS somente registra aquelas demandas que resultaram em estabelecimento de vínculos de trabalho, excluindo vagas de trabalho abertas (demandas) não preenchidas. Ademais, trata-se da demanda apenas por vínculos de trabalho contratados com indivíduos e, neste sentido, uma firma pode, por exemplo, demandar certas funções ou atividades através de contrato com outras firmas. Portanto, reitera-se aqui que a análise dos dados da RAIS é um proxy do comportamento 
da demanda das firmas de TIC no mercado formal de trabalho.

Por fim, cabe destacar a seguinte tendência no mercado de TIC: a presença dos chamados PJI, ou seja, pessoas jurídicas individuais. Trata-se de profissionais que individualmente se constituem como empresas através do registro de pessoa jurídica e normalmente prestam serviços de TIC no mercado; concorrendo, em alguns casos, com as demais empresas de TIC que possuem funcionários.

Uma maneira indireta e aproximada para compreender a representatividade dos $\mathrm{PJI}$ no setor de tecnologias da informação e comunicação é comparar o número de RAIS negativas com os demais estabelecimentos; pois, se a característica comum aos PJI é ser uma empresa sem vínculos de trabalho, estes devem fazer a declaração de RAIS negativa. Contudo, encontramos nessa declaração, por exemplo, estabelecimentos que suspenderam suas atividades naquele ano, ou seja, nem toda declaração de RAIS negativa é PJI. Além disso, podemos considerar PJI pequenas empresas que possuem funcionários, porém nenhum deles exerce ocupação ligada à atividade-fim da firma de TI. Portanto, o gráfico a seguir é apenas uma estimativa da representatividade dos PJI no setor de serviços e de serviços TIC:

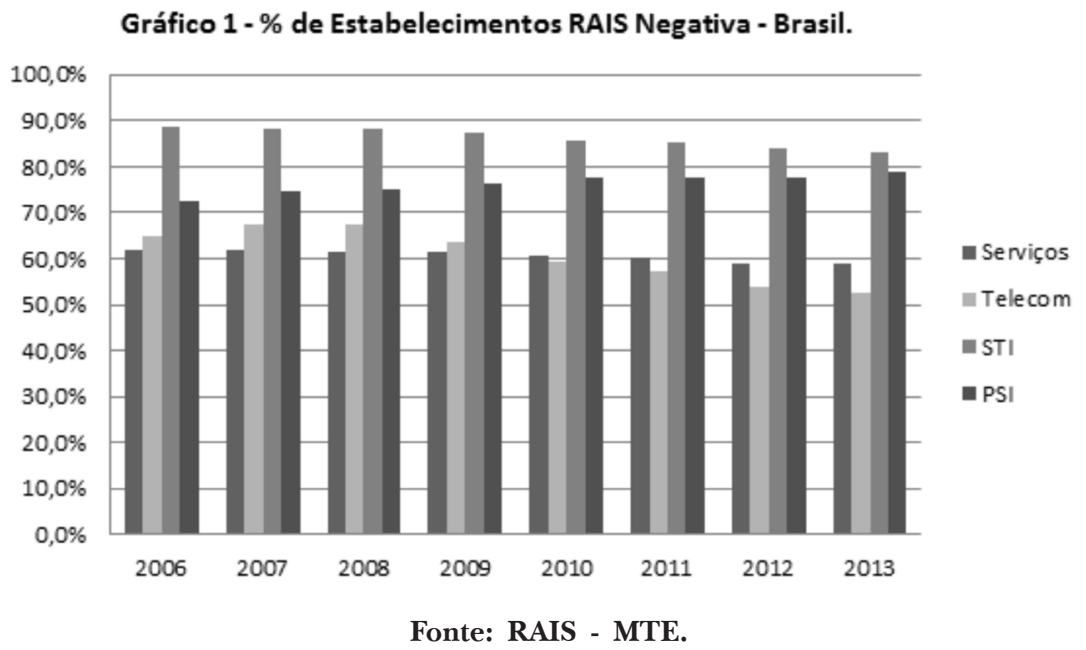

Para fins de clareza na exposição dos dados, adotamos as seguintes contrações ou siglas para os setores de atividades que estamos analisando: 1) o grande setor de serviços é denominado "Serviços"; 2) os serviços 
de telecomunicações aparecem como "Telecom"; 3) STI é a sigla para "Serviços de Tecnologia da Informação"; 4) PSI é "Prestação de Serviços de Informação".

As atividades de serviços TIC, com exceção das telecomunicações a partir de 2010, apresentam uma concentração maior de RAIS negativas do que no setor de serviços como um todo. Apesar de representarem uma grande parcela dos estabelecimentos, os PJI ou RAIS negativas não foram incluídos aqui na análise, porque, dada as próprias regras da base RAIS, a declaração negativa exime o estabelecimento de fornecer qualquer outra informação, ou seja, não há dados disponíveis nessa base sobre eles. Em razão do elevado número de declarações negativas, especialmente nos serviços TIC, compreendemos ser importante que futuros estudos enfoquem nesses "ilustres desconhecidos", que são os PJI e, de maneira mais ampla, a realidade dos estabelecimentos de declaração RAIS negativa.

Em suma, empreendemos uma análise estatística descritiva, que compara as divisões 61, 62 e 63 com o setor de serviços como um todo. Essa análise utiliza dados da base RAIS para o Brasil entre os anos de 2006 a 2013.

\section{A Demanda das Empresas de Serviços de TIC no Mercado de Trabalho}

Se a análise se estrutura na comparação dos serviços TIC com o grande setor de serviços, cabe começar aqui descrever o peso do número de estabelecimentos dos primeiros diante do último. 
Tabela 5 - Participação dos Estabelecimentos de Serviços TIC no Setor de Serviços - Brasil

\begin{tabular}{|c|c|c|c|c|}
\hline & Serviços & Telecom & STI & PSI \\
\hline \multirow{2}{*}{2013} & 1.393 .174 & 8.119 & 17.043 & 9.163 \\
\hline \multirow{2}{*}{2012} & $(100,0)$ & $(0,6)$ & $(1,2)$ & $(0,7)$ \\
\hline \multirow{2}{*}{2011} & 1.325 .839 & 7.078 & 16.236 & 9.400 \\
\hline \multirow{2}{*}{2010} & $(100,0)$ & $(0,5)$ & $(1,2)$ & $(0,7)$ \\
\hline \multirow{2}{*}{2009} & 1.273 .575 & 6.303 & 14.984 & 9.815 \\
\hline \multirow{2}{*}{2008} & $(100,0)$ & $(0,5)$ & $(1,2)$ & $(0,8)$ \\
\hline \multirow{2}{*}{2007} & 1.196 .122 & 5.407 & 13.711 & 9.975 \\
\hline \multirow{2}{*}{2006} & $(100,0)$ & $(0,5)$ & $(1,1)$ & $(0,8)$ \\
\hline & 1.130 .461 & 4.399 & 11.975 & 10.649 \\
\hline & $(100,0)$ & $(0,4)$ & $(1,1)$ & $(0,9)$ \\
\hline & $(1070.962$ & 3.696 & 10.685 & 11.751 \\
\hline & $(100,0)$ & $(0,3)$ & $(1,0)$ & $(1,1)$ \\
\hline
\end{tabular}

Fonte: RAIS - MTE.

Constata-se que a participação dos estabelecimentos de serviços TIC é pequena em relação ao setor de serviços como um todo. No entanto, é interessante destacar a expansão acima de 100\%, no período entre 2006 e 2013, no número de empresas de Telecom e STI. Nessas duas atividades observa-se crescimento superior ao setor de serviços em geral, fato que pode ser um indicativo da animação - mas ainda pouco expressiva em termos proporcionais - dos segmentos empresariais ligados à economia informacional do país. Com exceção do segmento de PSI, os outros dois segmentos apresentaram um crescimento constante ao longo do período. Essa tendência reflete-se, conforme gráfico 2, nas taxas desse crescimento do número de estabelecimentos. 
Gráfico 2 - Taxa de Crescimento Anual do No. de

Estabelecimentos - Brasil.

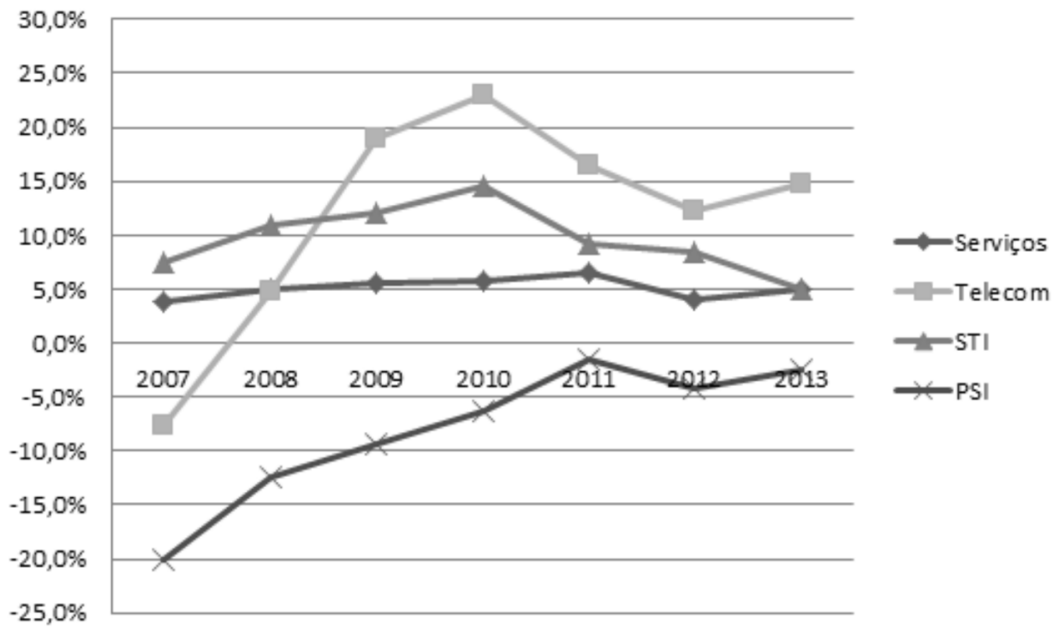

Fonte: RAIS - MTE.

O gráfico 2 mostra justamente uma redução do número de estabelecimentos PSI e taxas de crescimento ascendentes, em boa parte do período, no número de empresas de telecomunicações e de STI.

Se a participação dos números de estabelecimentos do setor de serviços TIC é pequena em relação ao grande setor; o que é possível observar com relação ao número de vínculos de trabalho demandado?

Tabela 6 - Participaço do No. de Vínculos de Trabalho de Estabelecimentos de Serviços TIC no Setor de Serviços - Brasil

\begin{tabular}{|c|c|c|c|c|}
\hline & Serviços & Telecom & STI & PSI \\
\hline \multirow{2}{*}{2013} & 37.396 .538 & 338.203 & 484.325 & 172.365 \\
\hline \multirow{2}{*}{2012} & $(100,0)$ & $(0,9)$ & $(1,3)$ & $(0,5)$ \\
\hline \multirow{2}{*}{2011} & 36.108 .264 & 314.950 & 476.238 & 172.828 \\
\hline \multirow{2}{*}{2010} & $(100,0)$ & $(0,9)$ & $(1,3)$ & $(0,5)$ \\
\hline & 34.701 .721 & 285.363 & 413.184 & 183.269 \\
\hline & $(100,0)$ & $(0,8)$ & $(1,2)$ & $(0,5)$ \\
\hline & 32.798 .399 & 226.117 & 396.450 & 190.663 \\
\hline
\end{tabular}




\begin{tabular}{|c|c|c|c|c|}
\hline & Serviços & Telecom & STI & PSI \\
\hline \multirow{2}{*}{2009} & 30.197 .654 & 201.253 & 334.276 & 185.324 \\
\hline \multirow{2}{*}{2008} & $(100,0)$ & $(0,7)$ & $(1,1)$ & $(0,6)$ \\
\hline \multirow{2}{*}{2007} & 29.219 .055 & 182.032 & 310.665 & 192.318 \\
\hline \multirow{2}{*}{2006} & $(100,0)$ & $(0,6)$ & $(1,1)$ & $(0,7)$ \\
\hline & 27.087 .985 & 175.562 & 253.026 & 203.567 \\
\hline & $(100,0)$ & $(0,6)$ & $(0,9)$ & $(0,8)$ \\
\hline & 25.332 .998 & 162.405 & 241.164 & 246.278 \\
\hline
\end{tabular}

Fonte: RAIS - MTE.

Também a participação da quantidade de vínculos de trabalho demandados pelos segmentos de serviços TIC é percentualmente baixa em relação ao setor de serviços. Em números de vínculos, o segmento de PSI encolheu em termos absolutos e na sua participação no grande setor. Porém, deve-se destacar que, da mesma forma que foi observado em relação ao número de estabelecimentos, o número de vínculos de trabalho em Telecom e STI cresceu a taxas maiores que o setor de serviços em geral, dando mostra de certa dinamização da economia informacional no país, em anos recentes. Telecom cresceu especialmente entre os anos de 2009 e 2011, auge da liberalização do mercado e abertura comercial do setor, mas apresentou certo arrefecimento após 2012. No segmento de STI constata-se uma taxa mais cíclica.

\section{Gráfico 3 - Taxa de Crescimento Anual dos Vínculos de Trabalho por Segmentos de Tl e Grandes Setores - Brasil}

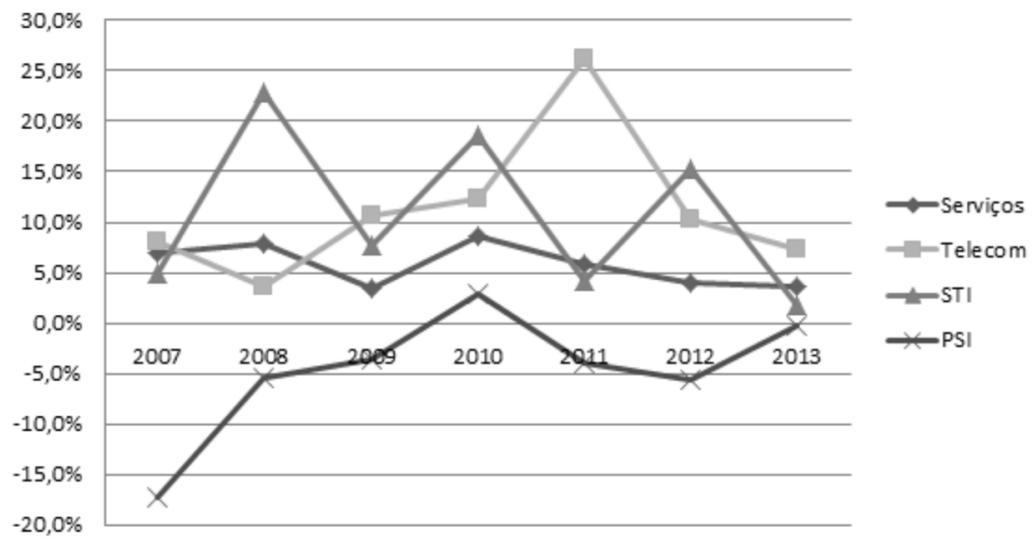

Fonte: RAIS - MTE. 
Mais uma vez, o segmento PSI apresentou taxas negativas de crescimento, exceto no ano de 2010. O crescimento dos outros dois segmentos de serviços de TIC manteve-se em taxas oscilantes ao se comparar o movimento ao longo do período: os STI, ao longo do período, apresentaram um movimento de ascensão e queda nas taxas, quando as comparamos anualmente; já o segmento de Telecom apresentou uma trajetória mais longa de taxas crescentes, mais especificamente entre os anos 2008 até 2011, e desde 2009 teve taxas de crescimento de contratações maiores que as do grande setor de serviços. Esse período em que há forte crescimento do número de vínculos de emprego na atividade de telecomunicações corresponde ao momento em que ocorre intensificação da concorrência no setor, quando as empresas iniciaram franca competição, baseada na convergência tecnológica. Após a liberalização do mercado, caracterizou-se um novo cenário empresarial em que as firmas visam a ampliar a participação no mercado por meio da competição, para que obtenham lucro e atraiam investidores. A concorrência depende de investimento em tecnologias, mudanças organizacionais, diversificação de produtos e serviços e empregados jovens e escolarizados, capacitados em fornecer suporte estratégico, operacional e técnico ao setor. Sob essas condições, ocorreu deslocamento na demanda por trabalho, que passou de especializado para qualificado.

Tanto o movimento dos indicadores sobre o número dos estabelecimentos como os de contratações indicam o estudo da relação entre as variações nesses indicadores e possíveis causas de retração ou crescimento do número de estabelecimentos e vínculos. Por exemplo, seria o movimento desses números determinados pelo desempenho macroeconômico brasileiro? Ou por variáveis institucionais, por exemplo, incentivos setoriais e carga tributária?

A base RAIS fornece poucas informações que dizem respeito à caracterização econômica da firma, centrando seus dados sobre as características da força-de-trabalho e dos vínculos. Todavia, é possível extrair uma descrição sobre o tamanho dos estabelecimentos e sua composição na demanda de trabalho. 


\section{Gráfico 4 - Distribuição de Estabelecimentos por Faixa de Vínculos de Trabalho - Brasil, 2013}

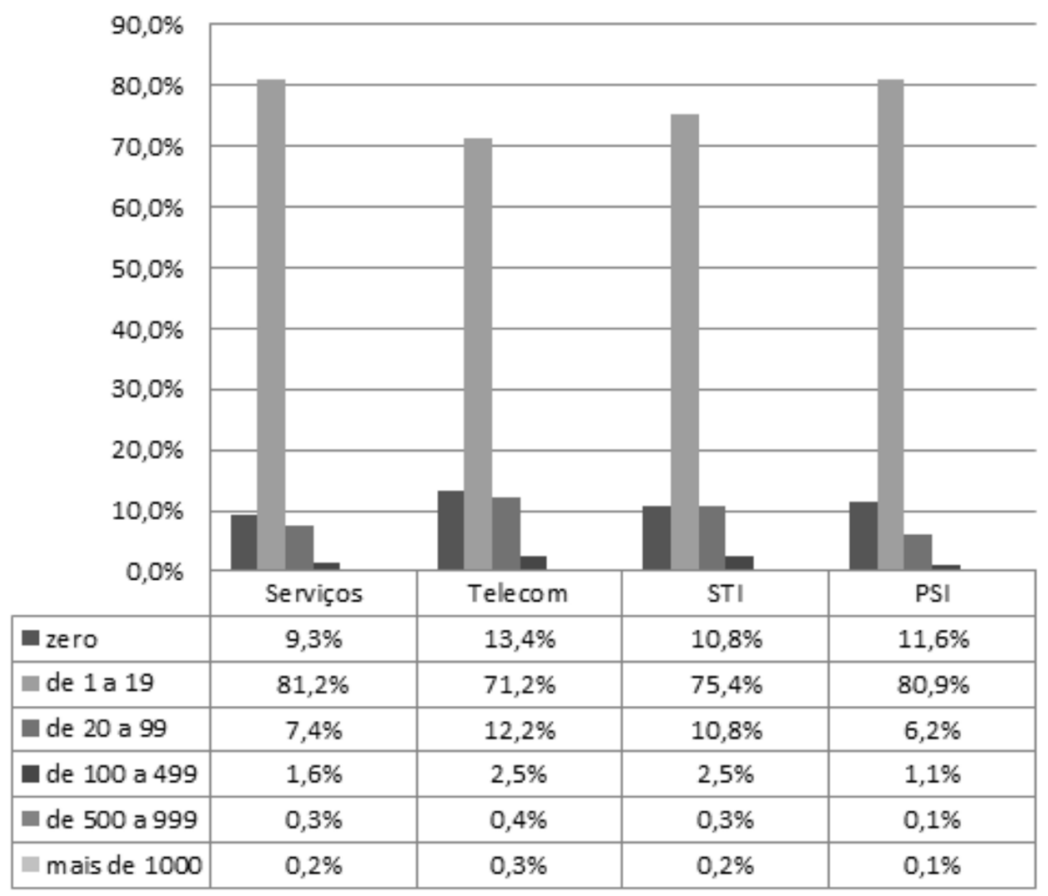

Fonte: RAIS - MTE.

Em ambos, no grande setor e nos três segmentos de TIC, a maior parte dos estabelecimentos declarou ter entre 1 e 19 vínculos de trabalho. Ao longo do período de 2006 a 2013, essa configuração não se modificou. Portanto, caberia concluir que o estudo do mercado de trabalho no setor de serviços e no de serviços de TIC precisa levar em conta que a grande maioria dos empregadores é de estabelecimentos de pequeno porte. Entretanto, é preciso identificar se a maior parcela das contratações ocorre por parte desses pequenos estabelecimentos. 


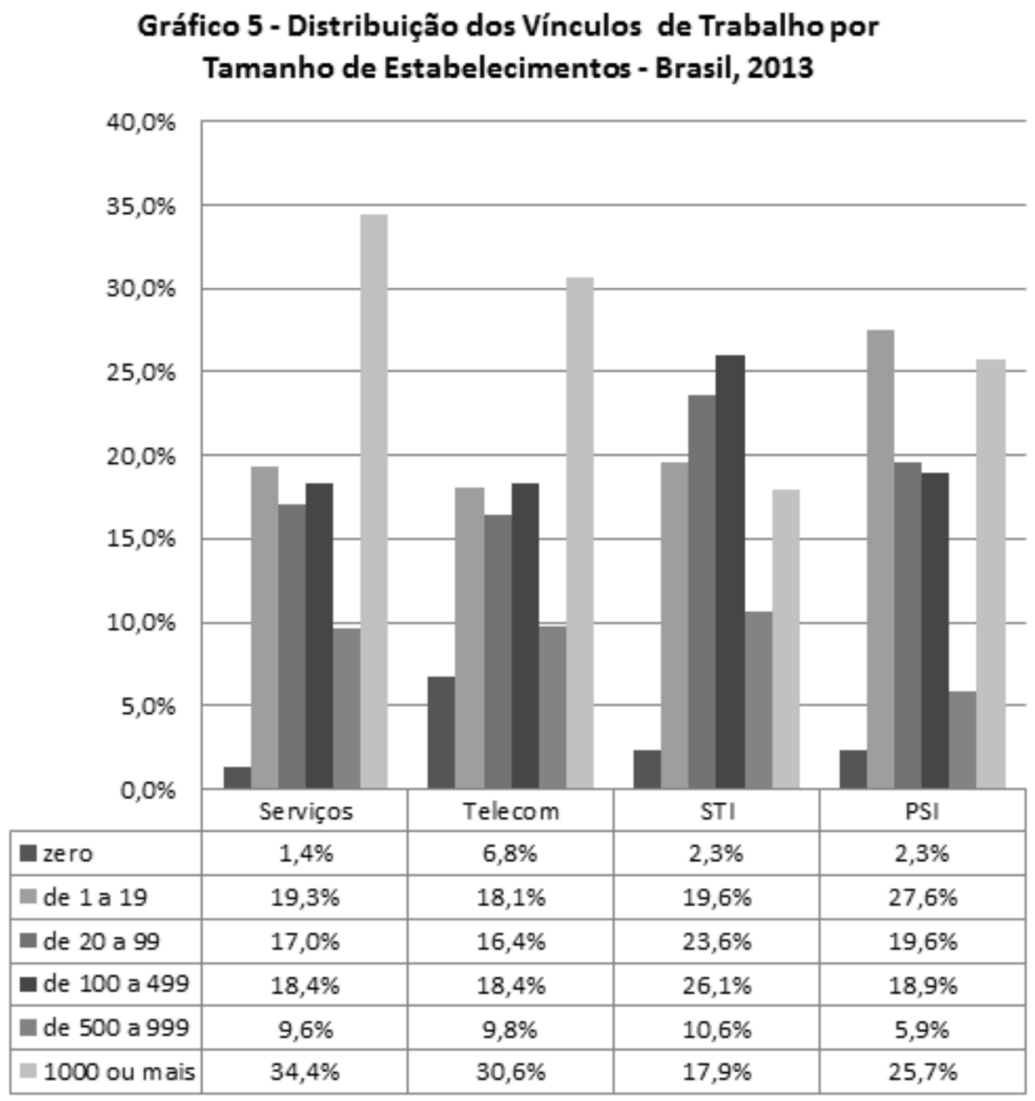

Fonte: RAIS - MTE.

Constata-se uma diferença entre, de um lado, o setor de serviços e o segmento de telecomunicações e, de outro, os segmentos STI e PSI. Enquanto nos primeiros há um maior predomínio de contratações nos grandes estabelecimentos, nos segundos há poucas diferenças entre os pequenos e os grandes estabelecimentos. Essa estrutura de distribuição não sofreu grandes alterações entre 2006 e 2013, e, portanto, pesquisas futuras precisam lidar com essas características da população de vínculos e estabelecimentos do mercado formal de trabalho dessas atividades. Por exemplo, será que a concentração de contratações nos maiores estabelecimentos indica pouca competição entre esses e os estabelecimentos médios e pequenos na hora de contratar mão de obra qualificada? Características dos vínculos de trabalho e do perfil da mão de obra contratada são diferentes quando consideramos 
setores com concentração de vínculos em maiores estabelecimentos comparando com os setores no quais os vínculos de trabalho estão mais significativamente nos estabelecimentos de menor porte?

Até aqui foi caracterizada a demanda de trabalho em serviços TIC pelo tamanho das firmas; o que se pode observar a respeito dos vínculos nessa demanda? Cabe destacar que a quase totalidade dos vínculos é CLT: tanto no setor de serviços como nos segmentos de serviços de TIC a porcentagem de vínculos CLT fica entre 97 e 99\%. O que, então, é possível verificar sobre a duração desses vínculos?

Tabela 7 - Média de tempo em meses dos vínculos de trabalho - Brasil

\begin{tabular}{|c|c|c|c|c|}
\hline & Serviços & Telecom & STI & PSI \\
\hline 2013 & 59,6 & 25,4 & 31,3 & 33,2 \\
\hline 2012 & 60,0 & 27,2 & 29,6 & 32,3 \\
\hline 2011 & 60,4 & 24,6 & 29,1 & 36,3 \\
\hline 2010 & 58,3 & 28,4 & 28,8 & 28,3 \\
\hline 2009 & 60,6 & 29,7 & 31,1 & 28,7 \\
\hline 2008 & 64,4 & 33,8 & 31,9 & 28,9 \\
\hline 2007 & 66,4 & 35,4 & 33,2 & 33,1 \\
\hline
\end{tabular}

Fonte: RAIS - MTE.

As empresas de serviços TIC apresentam médias de tempo de vínculo tradicionalmente menores das registradas no setor de serviços como um todo. Isso pode indicar que a "ida" das empresas TIC ao mercado de trabalho tem sido mais recorrente do que a de outras empresas do setor de serviços. Com exceção do segmento PSI, o setor de serviços, o segmento de telecomunicações e o de STI apresentaram redução nas médias de tempo ao longo do período. Cabe destacar esse aspecto porque, como foi demonstrado, o segmento PSI tem apresentado redução, ao longo de 2006 e 2013, do número de estabelecimento e de vínculos. Assim, como explicar essa redução combinada com um aumento na média da duração dos vínculos? Uma hipótese é que esteja relacionado a um núcleo de trabalho permanente com a existência de um entorno flexível. Porém, cabe investigar por que não ocorre nos segmentos centrais do setor de TIC e sim no segmento mais periférico. Deixamos em aberto essa questão para futuras pesquisas.

Essa diferença entre menores médias dos segmentos TIC em relação ao setor de serviços reflete nas diferenças entre as taxas anuais de não desligados: 
Tabela 8 - Participação (\%) de Vínculos Não Desligados - Brasil

\begin{tabular}{|c|c|c|c|c|}
\hline & Serviços & Telecom & STI & PSI \\
\hline 2013 & 69,6 & 60,8 & 67,3 & 63,5 \\
\hline 2012 & 69,5 & 62,0 & 66,2 & 62,6 \\
\hline 2011 & 70,4 & 63,9 & 67,3 & 63,7 \\
\hline 2010 & 70,8 & 65,5 & 64,5 & 63,9 \\
\hline 2009 & 72,9 & 67,4 & 67,1 & 63,6 \\
\hline 2008 & 71,5 & 70,4 & 63,1 & 62,5 \\
\hline 2006 & 74,3 & 70,2 & 67,8 & 62,0 \\
\hline
\end{tabular}

Fonte: RAIS - MTE.

Se o setor de serviços também apresenta porcentagens maiores de não desligados no ano, essa diferença em relação aos segmentos TIC não é tão maior quanto a verificada na comparação entre as médias de tempo dos vínculos. Novamente com exceção do segmento PSI, os demais apresentaram um movimento de redução da participação dos vínculos não desligados ao longo do período de 2006 a 2013.

O comportamento desses indicadores sobre a duração e estabilidade dos vínculos precisa ser explicado. Um ponto de partida possível é identificar se os vínculos estão sendo terminados por iniciativas das empresas (demissão) ou dos trabalhadores (desligamento).

Tabela 9 - Participação (\%) de Demissões e de Desligamentos nos Vínculos Terminados no Ano - Brasil

\begin{tabular}{|l|c|c|c|c|c|c|c|c|}
\hline & \multicolumn{2}{|c|}{ Serviços } & \multicolumn{2}{|c|}{ Telecom } & \multicolumn{2}{|c|}{ STI } & \multicolumn{2}{c|}{ PSI } \\
\cline { 2 - 10 } & Demissão & $\begin{array}{c}\text { Desliga- } \\
\text { mento }\end{array}$ & Demissão & $\begin{array}{c}\text { Desliga- } \\
\text { mento }\end{array}$ & Demissão & $\begin{array}{c}\text { Desliga- } \\
\text { mento }\end{array}$ & Demissão & $\begin{array}{c}\text { Desliga- } \\
\text { mento }\end{array}$ \\
\hline 2013 & 42,4 & 25,6 & 40,5 & 24,6 & 45,5 & 35,1 & 50,8 & 26,8 \\
\hline 2012 & 41,1 & 26,1 & 43,6 & 27,9 & 38,7 & 35,4 & 48,2 & 30,4 \\
\hline 2011 & 41,0 & 26,0 & 39,7 & 31,3 & 41,1 & 39,9 & 48,5 & 33,1 \\
\hline 2010 & 40,9 & 23,8 & 49,4 & 22,5 & 37,8 & 33,8 & 51,1 & 29,0 \\
\hline 2009 & 45,4 & 21,0 & 51,5 & 17,8 & 50,6 & 28,6 & 57,7 & 22,8 \\
\hline 2008 & 43,1 & 21,9 & 49,2 & 26,1 & 39,9 & 31,0 & 56,2 & 26,9 \\
\hline 2007 & 44,9 & 19,9 & 52,7 & 22,8 & 46,1 & 31,5 & 56,0 & 21,9 \\
\hline 2006 & 45,4 & 18,5 & 44,9 & 13,9 & 44,7 & 24,6 & 58,7 & 18,6 \\
\hline
\end{tabular}

Fonte: RAIS - MTE. 
Constata-se que no setor de serviços assim como nos segmentos de serviços TIC houve aumento da participação do término do vínculo por iniciativa do trabalhador. Isso pode indicar uma conjuntura na qual o mercado de trabalho apresenta um crescimento de oportunidades de emprego para os trabalhadores e um aumento da competição entre as empresas para contratação de mão de obra. Corroborando com essa hipótese, podemos observar que, exceto o segmento STI, todos apresentaram uma tendência de redução na participação das demissões nos vínculos terminados anualmente ao longo de 2006 a 2013. Entretanto, cabe destacar que as demissões ainda têm maior participação nos términos de vínculos, apesar do crescimento dos desligamentos. Por fim, podemos especular que a tendência de redução das médias de tempo dos vínculos de trabalho, assim como da menor participação dos vínculos não terminados, tem sido em parte causada por um movimento no qual os trabalhadores, por iniciativa própria, têm terminado o contrato de trabalho. Resta saber quais as causas que levaram ao aumento do número de trabalhadores que escolhem pedir desligamento da empresa.

Para contribuir com a verificação da hipótese de um mercado de trabalho dinâmico, com maiores oportunidades e incentivos para os trabalhadores mudarem de empresa, é relevante analisar o comportamento das médias salariais ao longo do período.

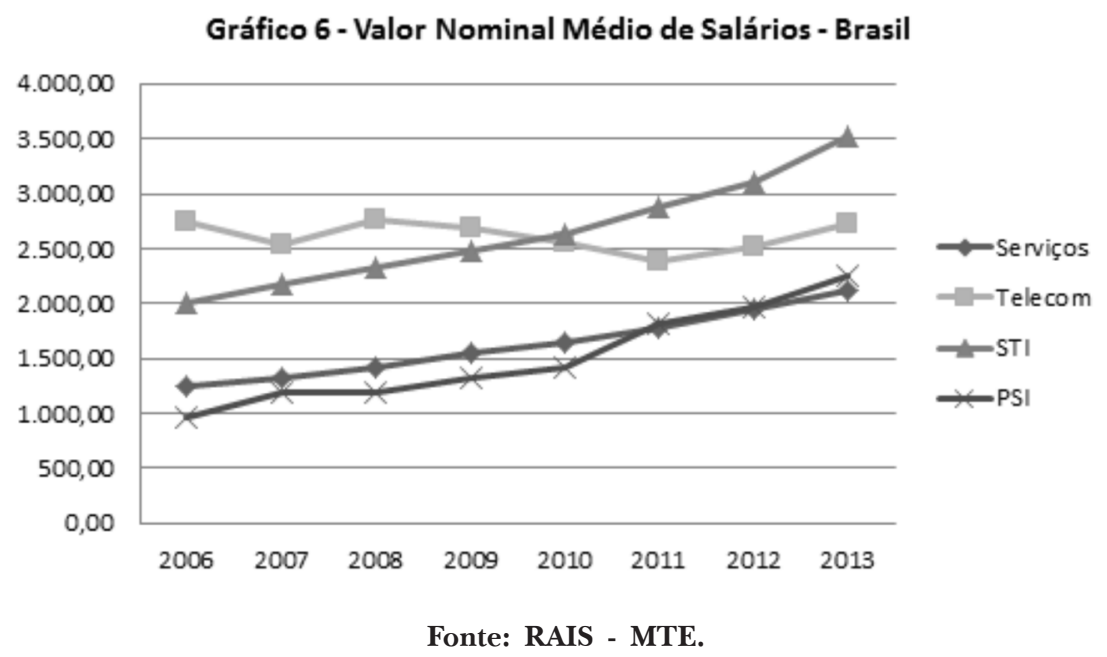

Exceto o segmento de telecomunicações, observa-se um aumento dos valores médios nominais dos salários, corroborando, portanto, com a ideia 
de um mercado de trabalho favorável aos trabalhadores. É curioso observar que o segmento de PSI apresenta crescimento na média salarial mesmo apresentando redução dos números de vínculos e de estabelecimentos no período (conforme tabelas 5 e 6 ). Todavia, quando analisamos as médias salariais em termos do número de salários mínimos, constata-se outra dinâmica:

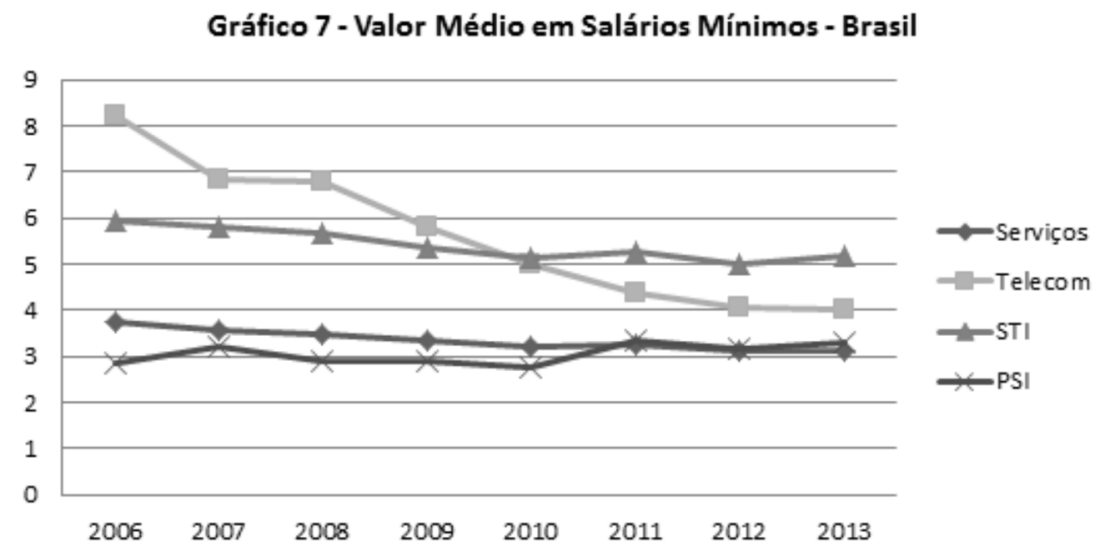

Fonte: RAIS - MTE.

Em termos de salários mínimos, as médias salariais do segmento de telecomunicações apresentam um movimento de queda acentuado e, com exceção do segmento PSI, nos demais, as médias se estabilizaram com uma leve tendência de queda. Talvez isso explique, então, o aumento da participação dos vínculos terminados pelos trabalhadores (conforme tabela 9). Uma vez que os ganhos nominais não excederam ou acompanharam os ganhos do salário mínimo, pode-se conjecturar que os trabalhadores desses segmentos de serviços de TIC e do setor de serviços procuraram oportunidades em outros segmentos da economia. No entanto, deve-se considerar a política governamental de aumento do salário mínimo em vigor no período. Enfim, é preciso maiores estudos para compreender as possíveis relações entre médias salariais e as características do número de vínculos e sua duração.

Apesar desse quadro no que se refere às médias salariais e a participação decrescente do número anual de não desligados, verificamos um aumento no número de contratações com exceção do segmento PSI. Todavia, o que podemos observar sobre as características dos trabalhadores que estão sendo 
demandados e contratos pelas empresas do setor de serviços e dos segmentos de serviços de TIC?

\section{Gráfico 8 - \% de Vínculos por Faixa Etária - Brasil, 2013}

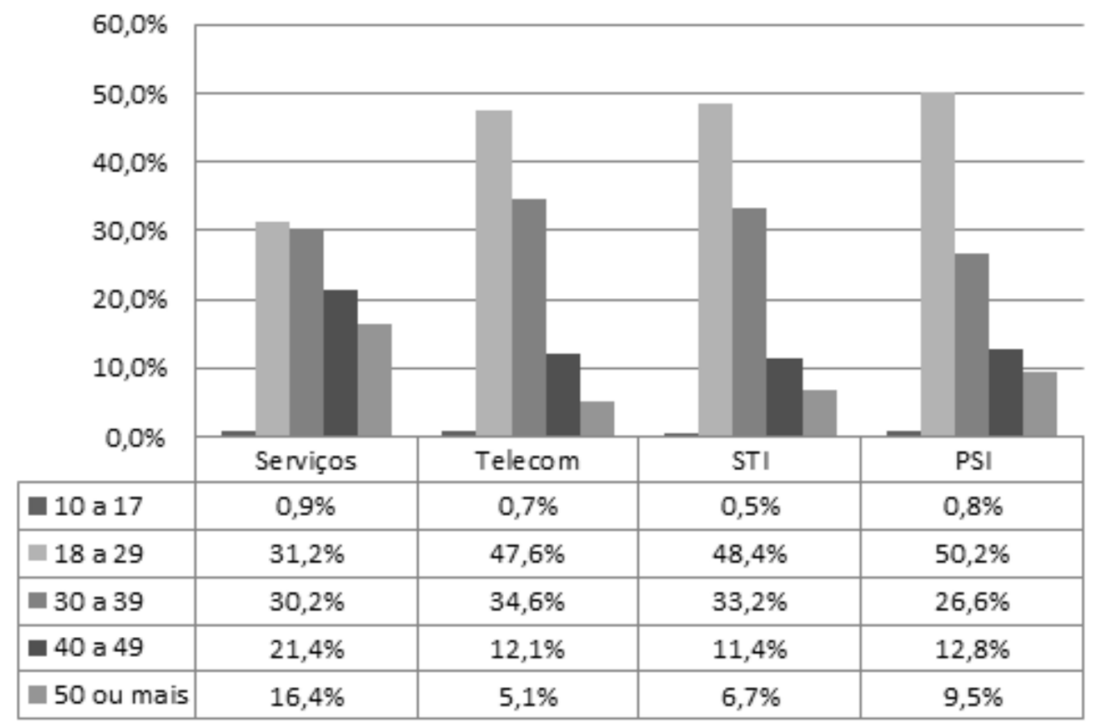

Fonte: RAIS - MTE.

Destaca-se que os segmentos de serviços TIC possuem uma concentração maior de vínculos de trabalho com trabalhadores mais jovens, especialmente na faixa entre 18 a 29 anos de idade. Talvez o fato de serem segmentos cujas atividades são marcadas pelo uso de novas tecnologias explique essa concentração de trabalhadores mais jovens. Outra explicação que pode ser aventada é a presença de uma preferência por mão de obra mais jovem por parte dessas empresas de serviços TIC.

Outro elemento importante para caracterizar a demanda de trabalho das firmas de TIC diz respeito ao nível de escolaridade da força de trabalho contratada. Será que os segmentos de serviços TIC, como atividades representativas de uma economia do conhecimento, demandam, em média, trabalhadores mais escolarizados? 
Gráfico 9 - \% de Vínculos por Escolaridade - Brasil, 2013

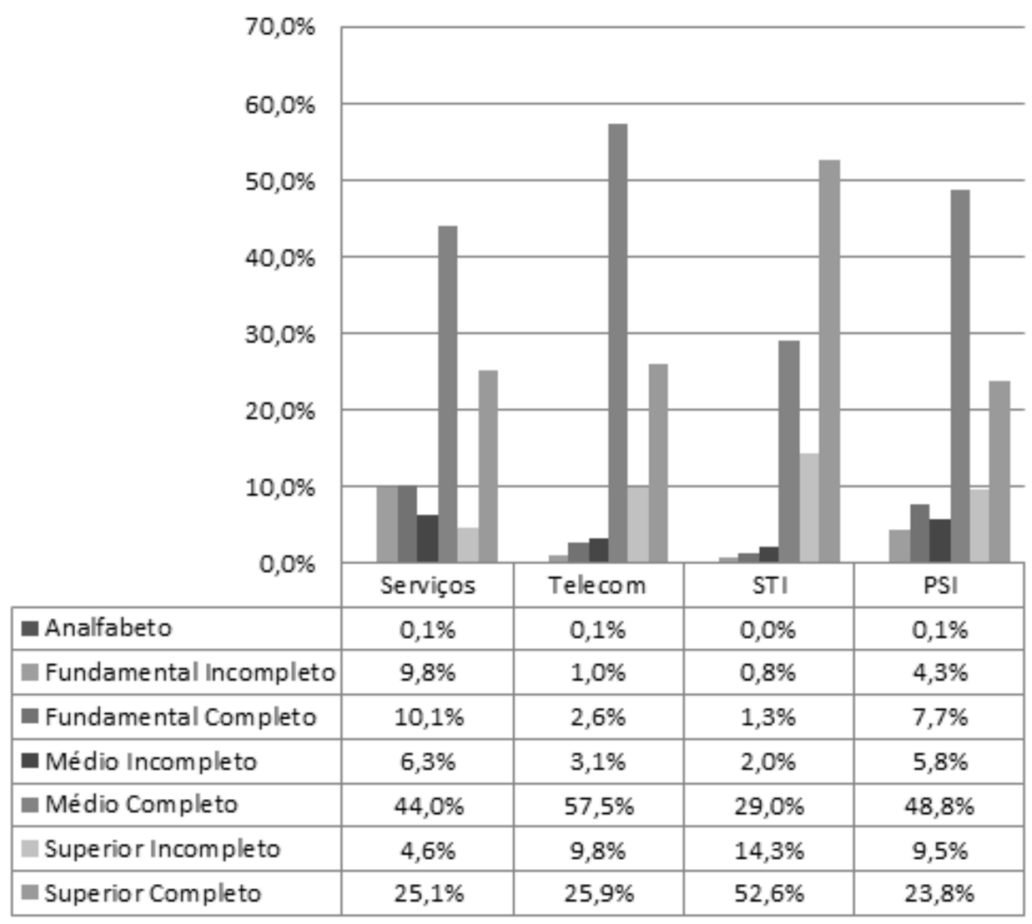

Fonte: RAIS - MTE.

Com exceção do segmento de STI, todos apresentaram maior concentração de contratações no nível de escolaridade "médio completo". Nesse sentido, o segmento de STI é bem peculiar e representativo da ideia de uma economia do conhecimento, pois pouco mais da metade das contratações ocorreu entre os trabalhadores com nível de escolaridade superior completo, e também são os que têm maior média salarial e maior concentração em faixa etária mais jovem. Essa diferença é um indicativo para não considerar o setor de serviços de TIC como uniforme, isto é, nesse setor é provável que existam realidades laborais e organizacionais bem distintas.

Podemos verificar no período uma tendência no aumento da participação nas contratações nos níveis "médio completo" e "superior completo"? 
Tabela 10 - \% de Vínculos de Trabalho Demandados com Curso Superior Completo - Brasil

\begin{tabular}{|c|c|c|c|c|}
\hline & Serviços & Telecom & STI & PSI \\
\hline 2013 & 25,1 & 25,9 & 52,6 & 23,8 \\
\hline 2012 & 24,0 & 24,4 & 46,1 & 20,9 \\
\hline 2011 & 23,4 & 26,0 & 45,3 & 20,8 \\
\hline 2010 & 22,8 & 30,9 & 45,8 & 16,7 \\
\hline 2009 & 22,9 & 33,5 & 40,4 & 14,7 \\
\hline 2008 & 22,2 & 36,8 & 38,7 & 13,9 \\
\hline 2006 & 21,8 & 33,8 & 38,8 & 14,9 \\
\hline
\end{tabular}

Fonte: RAIS - MTE.

Tabela 11 - \% de Vínculos de Trabalho Demandados com Ensino Médio Completo - Brasil

\begin{tabular}{|c|c|c|c|c|}
\hline & Serviços & Telecom & STI & PSI \\
\hline 2013 & 44,0 & 57,5 & 29,0 & 48,8 \\
\hline 2012 & 43,7 & 56,3 & 31,5 & 49,7 \\
\hline 2011 & 42,9 & 53,9 & 32,0 & 47,2 \\
\hline 2010 & 41,9 & 46,9 & 32,2 & 45,4 \\
\hline 2009 & 40,1 & 44,4 & 34,2 & 44,9 \\
\hline 2008 & 39,2 & 40,6 & 32,9 & 45,1 \\
\hline 2007 & 37,6 & 42,3 & 35,6 & 43,6 \\
\hline
\end{tabular}

Fonte: RAIS - MTE.

No caso do segmento de STI, constata-se uma clara redução da participação do nível "ensino médio completo" nas contratações em contraste com um forte crescimento da participação no nível "superior completo". Todavia, o setor de serviços e o segmento PSI apresentaram aumento na participação nas contratações no nível de escolaridade "superior completo", mas no segmento de telecomunicações verifica-se uma redução da participação desse nível de escolaridade combinado com um marcante crescimento da participação no nível "médio completo". Esses resultados corroboram com a ideia de que, dentro daquilo que podemos chamar de setor de serviços TIC, existem realidades laborais e organizacionais muito diversas.

Essas diferenças entre os segmentos de serviços TIC e o grande setor de serviços em termos da participação dos níveis de escolaridade "médio 
completo" e "superior completo" nas contratações do período analisado refletem-se na evolução das médias salariais desagregadas nesses dois níveis de escolaridade?

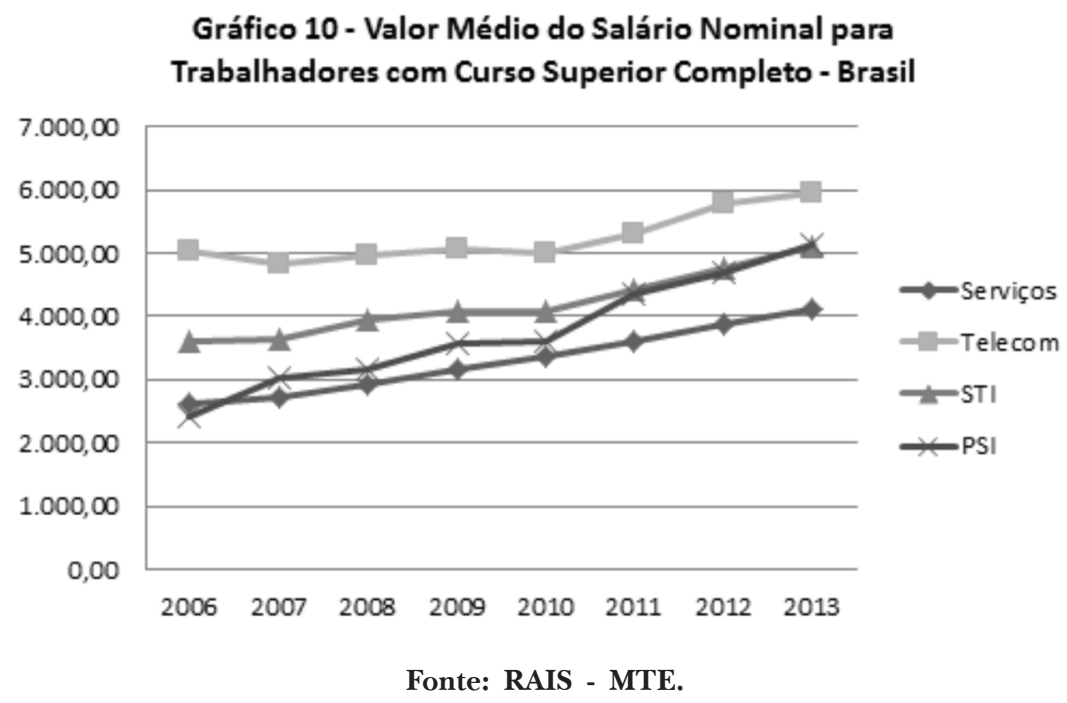

Gráfico 11 - Valor Médio do Salário Nominal para Trabalhadores com Ensino Médio Completo - Brasil

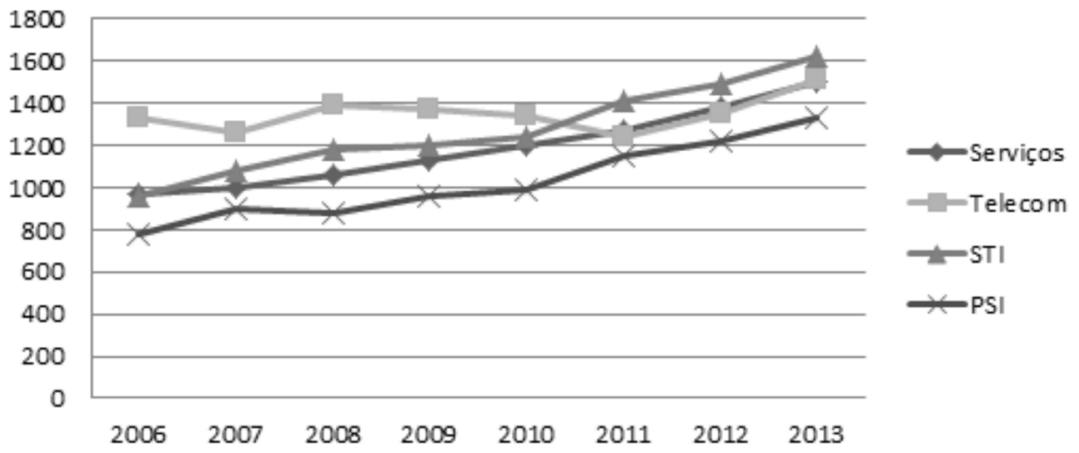

Fonte: RAIS - MTE.

Finalizando, nas médias salariais nominais, observa-se que em ambos os níveis de escolarização e em todos os segmentos de serviços TIC como, também, no grande setor de serviços, houve um movimento de aumento das médias salariais nominais durante o período de 2006 a 2013. No segmento de 
telecomunicações, enquanto a participação das contratações de trabalhadores com ensino superior completo diminui (ver Tabela 10), a média salarial para esse nível de escolarização não só aumentou como, também, foi a maior comparada com o grande setor de serviços e os demais segmentos de serviços TIC, durante todo o período analisado. Será que esse fenômeno é explicado pelo fato de que a redução da participação permitiu pagar salários mais altos para um número menor de trabalhadores? Ou, então, o aumento da média salarial combinado com a diminuição da participação das contratações de trabalhadores com ensino superior completo reflete que essa redução da participação não foi um movimento desejado e planejado pelas empresas de telecomunicações, que procuraram reverter essa redução aumentando os salários oferecidos aos trabalhadores com o nível de escolaridade em questão? Nesse sentido, cabe destacar que, enquanto a participação nas contratações dos trabalhadores com ensino médio completo diminui no segmento de STI, a média salarial aumentou de tal maneira que, em 2013, esse segmento apresentou a maior média comparado com o grande setor e os demais segmentos de serviços TIC. Portanto, essa relação entre médias salariais e participação das contratações por níveis de escolaridade precisa ser mais bem estudada para compreender o quanto ela é determinada por mudanças e decisões encaminhadas pelas empresas ou por outros fatores exógenos às firmas, por exemplo, políticas de emprego e renda.

\section{Considerações finais}

A principal constatação que este estudo de análise descritiva estatística alcançou é que dentro do que se pode chamar de serviços de TIC existem realidades laborais heterogêneas entre segmentos desse setor de serviços. Tal constatação corrobora com a tese mais geral de que, apesar de haver tendências gerais, as mudanças provocadas pela emergência do capitalismo informacional são complexas e diversas entre diferentes níveis de realidade socioeconômica. Pesquisas futuras precisam considerar esse ponto para evitar generalizações indevidas.

O setor de STI é o mais próximo da realidade de mercado típica do paradigma informacional, parece representar precisamente as características do comportamento da demanda de trabalho das firmas de ponta do ramo de TIC. Comparativamente, esse segmento é uma novidade em termos de mercado de trabalho no país: a expansão em termos de estabelecimentos 
e vínculos de trabalho indica a tendência identificada globalmente pela exigência da demanda por um perfil profissional qualificado e em grande parte altamente escolarizado, com predominância de pessoal com nível superior.

Telecom é um mercado com as peculiaridades de um setor que se reestruturou mediante as novas TIC, tem importância decisiva na economia atual, mas não chega a ser caso típico do paradigma informacional. Trata-se de um setor tradicional que se modernizou, adaptando-se ao novo ambiente de convergência tecnológica e franca concorrência de mercado. As firmas do setor demonstram ampliação da demanda por um perfil profissional qualificado e escolarizado. Ademais, houve crescimento da participação de profissionais com nível superior, mas é ampla a presença de pessoal de nível médio e técnico. É um setor em que não necessariamente as firmas criam novos produtos e serviços, sobretudo respondem à forte competição de mercado. O setor de Telecom é ativo no paradigma informacional e, portanto, a demanda por trabalho é a de um perfil profissional tecnicamente habilitado para armazenar, organizar e transmitir informação, que precisou se adequar às exigências de uma atividade econômica central para operacionalizar a nova economia.

PSI é um segmento de suporte e prestação de serviços aos variados setores produtivos da economia informacional, semelhante a um mercado de segunda classe. A oscilação negativa no número de estabelecimentos e de vínculos de emprego, as menores médias salariais, a reduzida permanência no emprego apontam para a caracterização de uma atividade produtiva que responde à flexibilidade funcional do mercado de trabalho. Mesmo com a diminuição de postos de trabalho, as firmas desta atividade econômica demonstram ampliação da demanda por um perfil profissional com nível superior, mas, tal como no setor de Telecom, é ampla a presença de pessoal de nível médio e técnico. O setor de PSI é passivo no paradigma informacional e por isso a demanda por trabalho é a de um perfil profissional capacitado a responder às exigências de uma atividade econômica assessória à lógica organizacional da nova economia. 


\section{Referências}

CASTELLS, Manuel.

(2000). A sociedade em rede. 4. ed. São Paulo: Paz e Terra.

CENTENO, Mário; MACHADO, Carla; NOVO, Álvaro.

(2007). A criação e destruição de emprego em Portugal. Boletim Económico. Lisboa, Banco de Portugal.

DE NEGRI, João Alberto; DE NEGRI, Fernanda; COELHO, Danilo; TURCHI, Lenita.

(2006). Tecnologia, Exportação e Emprego. In: DE NEGRI, João Alberto De

Negri; Fernanda De Negri; Danilo Coelho (Org.); Tecnologia, exportação e emprego. Brasília, IPEA.

DE NEGRI, João Alberto; SALERN0, Mario Sergio (Org.).

(2005). Inovações, padrões tecnológicos e desempenho das firmas industriais brasileiras. Brasília; IPEA.

EISCHEN, Kyle.

(2000). Information Technology: History, Practice and Implication for Development. Disponível em: htttp://escholarship.org/uc/ item/951709tx. Acesso em 20 de outubro de 2011.

FERRANTI, David de; PERRY, Guilhermo; LEDERMAN, Daniel; MALONEY, William.

(2002). De los recursos naturales a la economía del conocimiento: comercio y calidad del empleo. Banco Mundial, Estudios sobre América Latina y el Caribe.

HUALDE, Alfredo; SERRANO, Arcelia.

(2005). La calidad del empleo de asalariados con educación superior en tijuana y monterrey: Un análisis cuantitativo. Revista Mexicana de Investigación Educativa, v. 10, n. 25; p. 345-374. México D.F.
HUALDE, Alfredo; MICHELLI, Jordy.

(2009). Mercados de Trabalho nos Setor de Tecnologias da Informação e Comunicação no México. In: Sonia Guimarães (0rg.); Trabalho, Emprego e Relações Laborais em Setores Intensivos em Conhecimento: Brasil, México e Canadá. Porto Alegre: Editora da UFRGS.

INFANTE, Ricardo (Org.).

(1999). La calidad del empleo: la experiencia de los países latiniamericanos y de los Estados Unidos. Santiago-Chile: OIT / ETM.

\section{INSTITUTO BRASILEIRO DE GEOGRAFIA E} ESTATÍSTICA.

(2009). O Setor de Tecnologia da Informação e Comunicação no Brasil, 2003-2006. Disponivel em: http://biblioteca.ibge.gov. br/visualizacao/livros/liv41716.pdf. Acesso em 2 de dezembro de 2010.

SALDANHA, Roberto da Cruz.

(2006). Sociedade da Informação e Mercado de Trabalho: uma abordagem empírica sob a ótica das atividades econômica. Tese de mestrado apresentada à Escola Nacional de Ciências Estatísticas (ENCE) / Instituto Brasileiro de Geografia e Estatística (IBGE).

\section{Recebido em}

novembro de 2015

\section{Aprovado em}

julho de 2015 